\title{
Simultaneous arterial and venous thromboembolic events associated with COVID-19. A case report
}

\author{
Ashley Reed, ${ }^{1}$ Caroline Howard, ${ }^{1}$ Mohamed Barakat, ${ }^{1}$ Ali Navi ${ }^{2}$ \\ ${ }^{1}$ Emergency Department; ${ }^{2}$ Department of Vascular Surgery, Southend University Hospital NHS Foundation \\ Trust, Prittlewell Chase, Westcliff-on-Sea, England, United Kingdom
}

\begin{abstract}
Infections with COVID-19 can induce thrombophilia and increase the risk of thrombotic events. We describe a case of a 64years-old male who presents with intermediate-risk pulmonary embolism and brachial artery thrombosis secondary to an undiagnosed patent foramen ovale and COVID-19 infection. The patient was successfully treated with thrombolysis and heparin infusions and was discharged home with long-term anticoagulation. Managing patients with multiple pathologies occurring concurrently requires effective inter-speciality working in order to take a holistic approach to managing the patient opposed to managing the conditions in isolation. This case report highlights how clinical guidelines can support decision making of the individual pathologies but working as a team will enable the best care.
\end{abstract}

Correspondence: Ashley Reed, Emergency Department, Southend University Hospital NHS Foundation Trust, Prittlewell Chase, Westcliff-on-Sea, SS0 0RY, England, United Kingdom.

E-mail: Ashleyreed@nhs.net

Key words: Pulmonary embolism; COVID-19; arterial embolism; patent foramen ovale; venous embolism; case report.

Contributions: AR, MB, AN: Managed the patient, devised the manuscript and the revisions; $\mathrm{CH}$ : Input into the manuscript and revisions

Conflict of interest: None. This work was not supported by any grant.

Availability of data and materials: All data generated or analyzed during this study are included in this published article.

Ethics approval and consent to participate: Ethics approval is not required at our institution to publish an anonymous case report. Informed consent was obtained from the patient included in this study.

Consent for publication: Informed consent gained from the patient.

Received for publication: 1 March 2021.

Revision received: 14 April 2021.

Accepted for publication: 20 April 2021.

This work is licensed under a Creative Commons Attribution 4.0 License (by-nc 4.0).

${ }^{\circ}$ Copyright: the Author(s), 2021

Licensee PAGEPress, Italy

Emergency Care Journal 2021; 17:9718

doi:10.4081/ecj.2021.9718

\section{Case Report}

A 64-years-old black African male had been complaining of left arm pain for three days. He had been prescribed naproxen and lansoprazole by his General Practitioner (GP) on day two for suspected musculoskeletal pain or diabetic neuropathy given that reduced sensation to $\mathrm{C} 5 / \mathrm{C} 6$ dermatome was noted. On the third day of symptoms, he suddenly became short of breath then collapsed, when walking from his car to the GP surgery. An emergency ambulance was called, they provided 15 litres per minute of oxygen and pre-alerted him to the Emergency Department (ED).

At our evaluation in the ED the patient was alert, dyspnoeic with tachypnoea (respiratory rate of 28), oxygen saturation was unrecordable, pulse rate of $128 \mathrm{bpm}$ and blood pressure of $154 / 96 \mathrm{mmHg}$. The observations made him score 10 on the National Early Warning Score. He was recently diagnosed with type two diabetes and had a blood glucose of $13.4 \mathrm{mmol} / \mathrm{L}$. He was prescribed and compliant with naproxen, lansoprazole and metformin.

On examination, his left upper limb was cold and pale with slight reduction in the sensation of the hand. The axillary pulse was palpable, but nil further was palpable distally. The patient's chest was clear.

Given this history and clinical findings suggestive of a Pulmonary Embolism (PE) and potential left upper limb arterial thrombosis an arterial blood gas was obtained from his right radial artery and a Computed Tomography Pulmonary Angiogram (CTPA) and left upper limb angiogram was requested (Figure 1 and 2). The intensive care team and vascular surgeon were alerted for their specialist input.

The ABG demonstrated a significant type one respiratory failure $\left(\mathrm{PaO}_{2}\right.$ of $6.36, \mathrm{FiO}_{2}$ of 0.85$)$, a compensatory respiratory alkalosis ( $\mathrm{pH} 7.42, \mathrm{PaCO}_{2}$ of 4.17) secondary to a metabolic lactic acidosis $\left(\mathrm{HCO}_{3}^{-}\right.$20.1, Lactate 2.28).

The electrocardiogram showed a sinus tachycardia which is the most common rhythm seen in acute PE. ${ }^{1}$

Blood tests including full blood count, urea and electrolytes, coagulation studies and a blood culture were obtained. D-dimer test was requested only for its prognostic role in COVID-19 patients, as its use in the diagnostic algorithm of PE was not indicated (pre-test probability of 4.5 using the Wells' Criteria for PE). ${ }^{2}$ The result did not affect the treatment decision and it was elevated at $9526 \mathrm{mcg} / \mathrm{L}$. Some evidence is emerging regarding thresholds for d-dimer that are strongly indicative of PE in COVID-19 patients $(>2660 \mathrm{mcg} / \mathrm{L}$ having $100 \%$ sensitivity and $67 \%$ specificity). ${ }^{3}$ The renal function had declined [creatinine 155 (baseline 95), urea 13.0 (baseline 5.7)], demonstrating an Acute Kidney Injury (AKI) stage one.

CTPA, completed after 126 minutes in the ED due to departmental radiology delays, confirmed left and right main pulmonary artery emboli extending into the segmental branches of all lobes and a dilated 
right atrium and flattening of the interventricular septum consistent with right heart strain. There was a bulging at the left side of the interatrial septum, which raised the suspicion of a patent foramen ovale. The CTPA also highlighted peripheral ground glass opacities consistent with moderate/severe COVID-19 infection.

Left upper limb angiogram confirmed a brachial artery embolus extending into the radial artery.

A polymerase chain reaction swab later confirmed the suspicion of COVID-19 infection.

The initial treatment for the type one respiratory failure was oxygen, which was provided at 15 litres per minute through a tightfitting mask with reservoir. This achieved arterial oxygen saturations of only $80 \%$. Given the fluid losses through the increased ventilatory rate and AKI, sodium chloride was administered.

Risk stratification and guidance on treatment for PE with COVID-19 infection do not exist. However, factors such as patient location, disease severity, d-dimer thresholds and clinical features of venous thromboembolism should all be considered to make decisions regarding treatment as suggested by the British Thoracic Society. ${ }^{4}$ We judged the disease state as severe due to the high oxygen requirements $\left(\mathrm{PaO}_{2}\right.$ of $6.36, \mathrm{FiO}_{2}$ of 0.85$)$, the signs of right heart strain and the concomitant arterial thrombosis. This patient's Pulmonary Embolism Severity Index (PESI) score was 134 which is classed as very high risk and has an associated 30-day mortality of $10-24.5 \% .^{5}$

Considering the acutely ischaemic left upper limb as an isolated pathology, the most definitive treatment option would have been open or endovascular embolectomy. However, including the more challenging pathology, PE and COVID-19, the benefit of medical management overrode the surgical approach. Therefore, the patient was treated with systemic thrombolysis (alteplase in this case) for both the PE and arterial thrombus, after 189 minutes in the ED. The dose of alteplase was $0.9 \mathrm{mg} / \mathrm{kg}$, which was $81 \mathrm{mg}$ of alteplase given as $10 \%$ ( $8 \mathrm{mg}$ bolus over 2 minutes) followed by $90 \%$ (73mg) infusion over 1 hour. Thirty minutes after the alteplase infusion a maintenance dose intravenous unfractionated heparin at $1 \mathrm{~mL} / \mathrm{hr}$ (1000 units/hour) was administered.

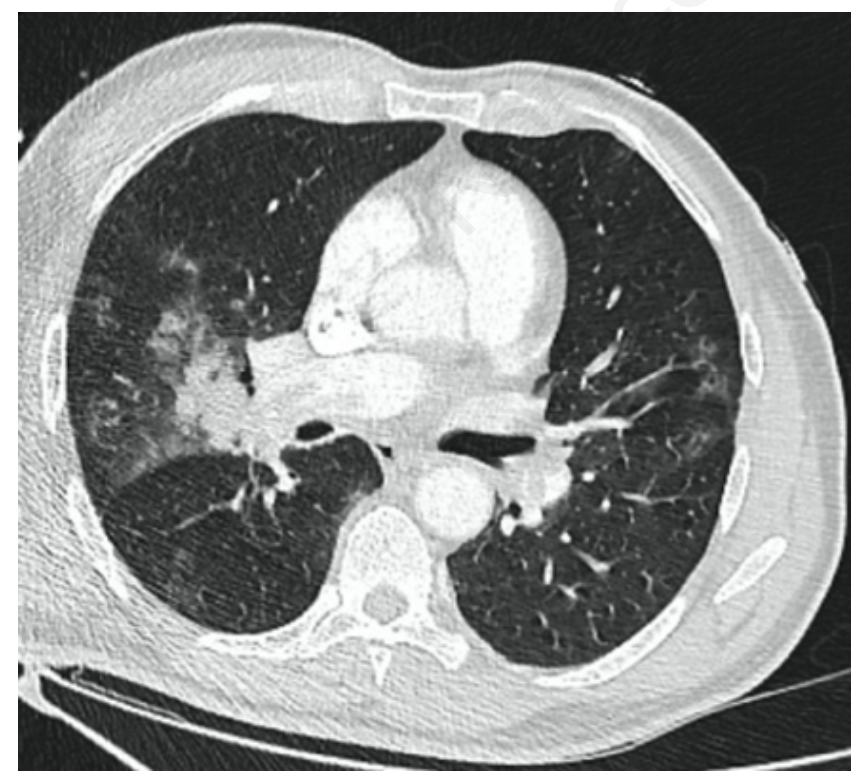

Figure 1. Computed tomography pulmonary angiogram demonstrating pulmonary emboli and appearances suggestive of COVID-19 infection.
One hour after the thrombolytic infusion had finished the patient was titrated off oxygen and maintained oxygen saturations of $98 \%$. He reported his limb was starting to feel normal again and clinically had a better capillary refill time.

The patient was managed on an acute respiratory ward and discharged home on day 5 with rivaroxaban for long term anticoagulation. He was followed up with the respiratory team as an outpatient. An echocardiogram performed two months after discharge highlighted a shunt across the atrial septum (Figure 3).

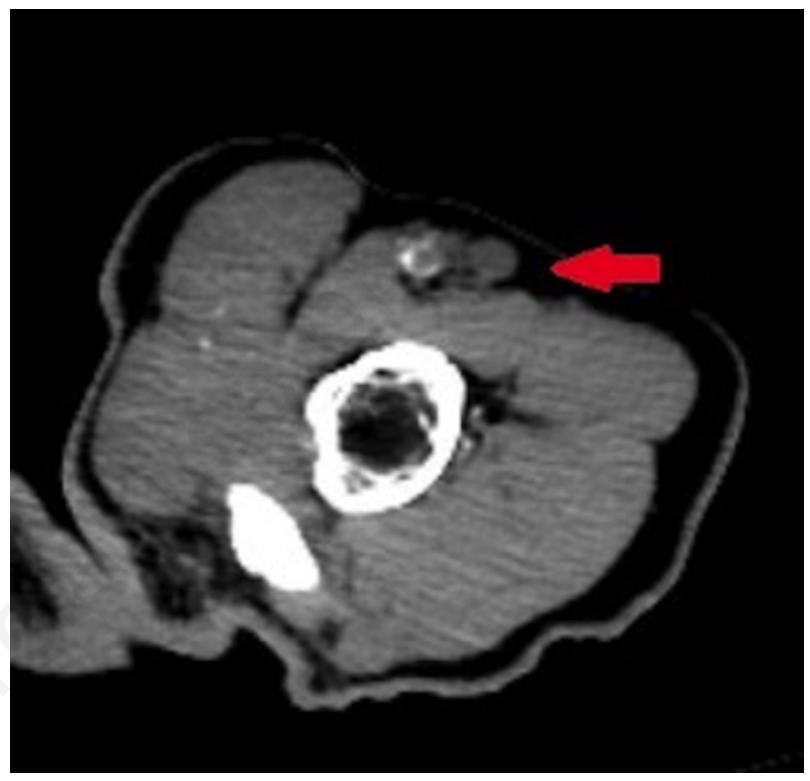

Figure 2. Upper limb angiogram demonstrating embolus in brachial artery

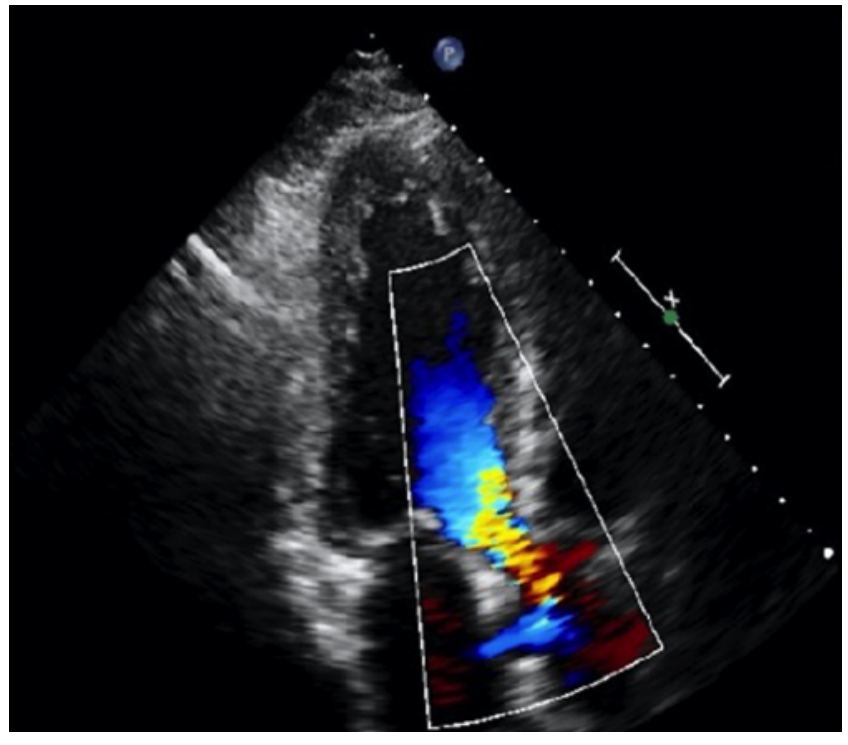

Figure 3. Echocardiogram demonstrating shunt across atrial septum. 


\section{Discussion}

The incidence of thrombosis in COVID-19 pneumonia patients has been highlighted to be $49 \%$ in three Dutch intensive care units, of which $87 \%$ were PEs. ${ }^{6}$ These thrombotic complications associated with COVID-19 are thought to be due to the cytokine storm that occurs with the infection which subsequently increases the clotting factors, notably fibrinogen. ${ }^{7}$ However, the patient's fibrinogen in this case was within the normal limits at $4.65 \mathrm{~g} / \mathrm{L}$ at admission. Moreover, the other prognostic markers of COVID-19 infection, C-reactive protein $(18 \mathrm{mg} / \mathrm{L}$ where normal is $<5)$, lymphopenia, $\left(1.05 \times 10^{9} / \mathrm{L}\right)$ and liver enzymes (only globulin 40 where normal is $20-35 \mathrm{~g} / \mathrm{L}$ ) were not pronounced to indicate severe COVID-19 infection.

It has been found in a study of 183 patients with severe COVID-19 infections that d-dimers with a median level of $2,120 \mathrm{mcg} / \mathrm{L}$ occurred in non survivors and $610 \mathrm{mcg} / \mathrm{L}$ in survivors. ${ }^{8}$ This patient had a d-dimer of $9526 \mathrm{mcg} / \mathrm{L}$ indicating that survival may be compromised by the inflammatory/thrombotic state. The ddimer is non-specific, and it cannot differentiate between inflammation and thrombosis, and in this case the patient's other markers of inflammation were not significantly raised thus it may be that the elevated d-dimer in this case was secondary to thrombosis as opposed to systemic inflammation.

Surprisingly this patient did not present with cough, fever or myalgia preceding the limb ischaemia but had significant changes on CTPA to evidence moderate to severe COVID-19 infection. Therefore, the diagnosis of COVID-19 came after the thrombotic complications had manifested. It should also be noted that this was an otherwise unprovoked PE, as the patient otherwise had no risk factors for thromboembolism. In addition, the Sepsis Induced Coagulopathy (SIC) score was 2 meaning that SIC is unlikely yet still carries a mortality risk at 28 days of $20 \% .{ }^{9}$ The low SIC score further supports a thromboembolic disease process rather than overt coagulopathy secondary to sepsis as a cause.

The use of thrombolysis for patients with high-risk PE defined as obstructive shock, sustained hypotension or cardiac arrest is supported by the evidence..$^{10}$ However, evidence to support the use of thrombolysis in patients with intermediate-risk PE defined as $\mathrm{PE}$ with right ventricular dysfunction and/or raised cardiac biomarkers in the absence of shock, as in this case, is limited. ${ }^{11}$ Intermediate-risk PEs are always based on a case-by-case basis, given the associated upper limb thrombus and high PESI score the collaborative decision was to thrombolyse.

\section{Conclusions}

COVID-19 infections have been associated with thrombotic events, this can include both PE and arterial thrombus as in this case. Management of such cases can be supported by clinical guidelines for the individual pathologies. However, when multiple pathologies exist as in this case the inter-speciality teams must work cohesively in order to manage the patient holistically for the best outcomes.

\section{References}

1. Petruzzelli S, Palla A, Pieraccini F, et al. Routine electrocardiography in screening for pulmonary embolism. Respiration 1986;50:233-43.

2. Zhang L, Yan X, Fan Q, et al. D-dimer levels on admission to predict in-hospital mortality in patients with Covid-19. J Thromb Haemost 2020;18:1324-9.

3. Leonard-Lorant I, Delabranche X, Severac F, et al. Acute pulmonary embolism in COVID-19 patients on CT angiography and relationship to D-dimer levels. Radiology 2020:201561.

4. BTS Guidance on venous thromboembolic disease in patients with COVID-19. Updated 4 May 2020.

5. Aujesky D, Obrosky DS, Stone RA, et al. Derivation and validation of a prognostic model for pulmonary embolism. Am J Resp Critical Care Med 2005;172:1041-6.

6. Klok FA, Kruip MJ, Van Der Meer NJ, et al. Confirmation of the high cumulative incidence of thrombotic complications in critically ill ICU patients with COVID-19: an updated analysis. Thromb Res 2020;191:148-50.

7. Wise J. Covid-19 and thrombosis: what do we know about the risks and treatment? BMJ 2020;369:m2058.

8. Tang N, Li D, Wang X, Sun Z. Abnormal coagulation parameters are associated with poor prognosis in patients with novel coronavirus pneumonia. J Thromb Haemost 2020;18:844-7.

9. Iba T, Di Nisio M, Levy JH, et al. New criteria for sepsisinduced coagulopathy (SIC) following the revised sepsis definition: a retrospective analysis of a nationwide survey. BMJ Open 2017; 7:e017046.

10. Konstantinides SV, Meyer G, Becattini C, et al. 2019 ESC Guidelines for the diagnosis and management of acute pulmonary embolism developed in collaboration with the European Respiratory Society (ERS) The Task Force for the diagnosis and management of acute pulmonary embolism of the European Society of Cardiology (ESC). Eur Heart J 2020;41:543-603.

11. Meyer G, Vicaut E, Danays T, et al. Fibrinolysis for patients with intermediate-risk pulmonary embolism. N Engl J Med 2014;370:1402-11. 